\title{
DRUG-INDUCED ROWELL SYNDROME IN A MALE PATIENT
}

\author{
Vessel Kantardjiev $^{1}$, Elena Stoikova ${ }^{1}$, Valentina Broshtilova ${ }^{2}$ \\ ${ }^{1}$ Department of Dermatology and Venereology, Military Medical Academy, Sofia, Bulgaria \\ ${ }^{2}$ Department of Infectious Diseases, Parasitology and Dermatovenereology, \\ Faculty of Medicine, Medical University of Varna
}

\begin{abstract}
Rowell syndrome is a rare skin disease described as an association of erythema multiforme and systemic lupus erythematosus, immunologically characterized by speckled antinuclear, anti-Ro, and anti-La antibodies. The majority of described cases affect middle-aged women. We report a case of a 53-year-old man with no previous history of lupus erythematosus, who developed erythema multiforme-like lesions and immunological findings consistent with Rowell syndome upon an intake of non-steroidal anti-inflammatory drugs. The patient responded well to systemic steroids and hydroxychloroquine. A short overview, emphasizing the specific clinical, laboratory and histology findings of this peculiar syndrome, is also presented.
\end{abstract}

Keywords: Rowell syndrome, lupus erythematosus, erythema multiforme

\section{INTRODUCTION}

Scholtz first described the association between lupus erythematosus (LE) and erythema multiforme (EM) in 1922 (1). In 1963 Rowell et al. reported a rare distinctive entity, characterized by discoid lupus and EM-like lesions, positive rheumatoid factor, speckled antinuclear antibody, and precipitating antibody to saline extract of human tissue (2). In 2000, Zeitouni and coworkers suggested major (coexistence of LE,

\footnotetext{
Address for correspondence:

Valentina Broshtilova

Faculty of Medicine

Medical University of Varna

55 Marin Drinov St

9002 Varna

e-mail:broshtolova@mail.bg
}

Received: November 13, 2018

Accepted: December 9, 2018
EM-like lesions and speckled ANA pattern) and minor (chillblains, positive RF, and anti-Ro or antiLa antibodies) diagnostic criteria (3). Up to date, only 12 male patients with Rowell syndrome have been reported worldwide $(4,5)$.

Herein, we report a case of drug-induced Rowell syndrome in a male patient.

\section{CASE REPORT}

A 53-year-old Caucasian man was admitted to our Department with a 2-month history of painful blisters and erosions on photo-exposed areas together with extended oral ulcerations. The skin lesions appeared upon a common cold treated with anti-inflammatory drugs - ibuprofen, paracetamol and metamizol. He had no previous medical history. No concomitant medications were taken.

Skin examination revealed significant erythema, edema, annular target-like lesions with blister formation and central necrosis, symmetrically disturbed on the sun-exposed areas (Fig. 1). Multiple 
small ulcers over extended enanthema were seen on hard palate and buccal mucosa. Skin biopsy revealed necrotic epidermis, vacuolar degeneration of the basal keratinocytic layer, and obscured dermo-epidermal junction by dense mixed inflammatory infiltrate intermingled with few eosinophils (Fig. 2). The direct immunofluorescence from an erythema multiforme-like lesion was negative.

Laboratory studies showed anemia $(\mathrm{Hb}-109 \mathrm{~g} / \mathrm{L})$, increased erythrocyte sedimentation rate $(87 \mathrm{~mm} / \mathrm{h})$, elevated C-reactive protein test $(7.7 \mathrm{mg} / \mathrm{L})$ and elevated functional liver tests (ASAT 98.3 U/L; ALAT 51.5 U/L; GGT-87.0 U/L).

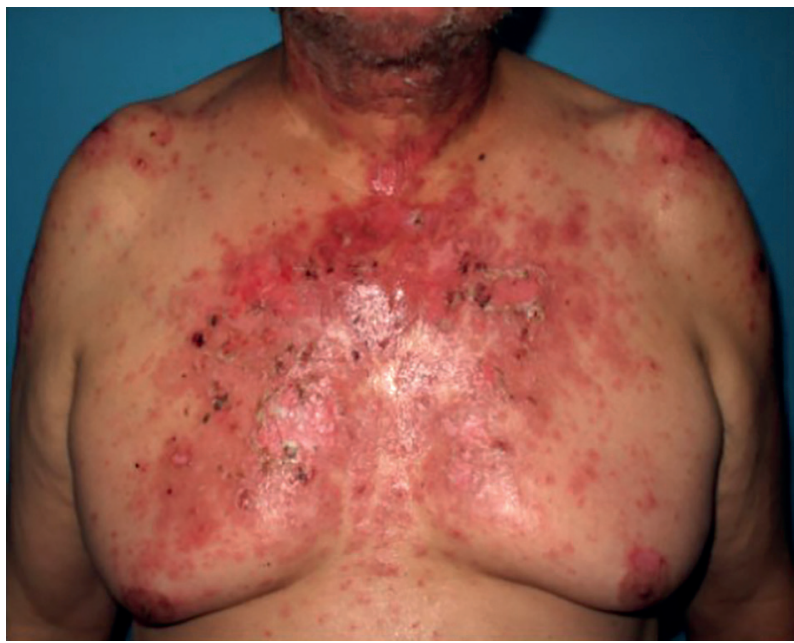

Fig. 1. Targetoid erythematous lesions with superficial blisters covered with hemorrhagic crusts on the trunk of the patient

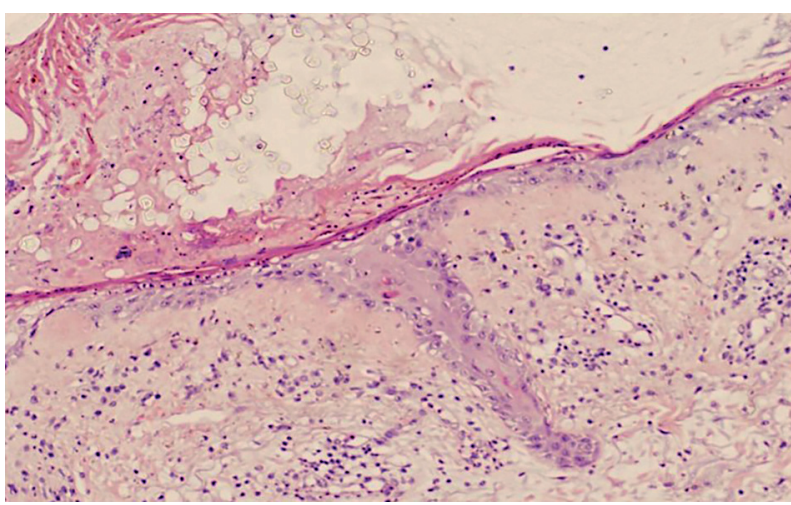

Fig. 2. Necrotic epidermis, vacuolar degeneration of the basal keratinocytic layer, mild interstitial and perivascular lympho-plasmocytic infiltration in the papillary dermis. Histological changes consistent with erythema multiforme (HE, $x$ 200)
Autoimmune screening showed highly elevated ANA (1:1280), increased levels of anti-ds DNA (>200) and positive levels of anti-Ro (anti-SSA) and anti-La (anti-SSB) antibodies. The complement fragments were decreased (C3 - $61 \mathrm{mg} / \mathrm{dL}$ and $\mathrm{C} 4-20 \mathrm{mg} / \mathrm{dL})$. The rheumatoid factor was negative.

Nail fold capillaroscopy revealed spastic cramp in the microcirculation of both hands. The chest Xray, electrocardiogram and abdominal ultrasound were normal.

Based on the clinical, histopathological, laboratory and immunological findings, the diagnosis of drug-induced Rowell syndrome was established. Intravenous methylprednisolone (80 $\mathrm{mg}$ per day), oral chloroquine phosphate (500 mg per day) and topical clobetasol propionate ointment were initiated with fast improvement, which allowed the discontinuation of corticosteroids in one month. At that point the skin lesions showed complete resolution with mild residual poikiloderma (Fig. 3). The patient was maintained on low-dose oral choroquine phosphate for 6 months. No recurrence occurred during this follow-up period.

\section{DISCUSSION}

Rowell syndrome is a rare presentation of lupus erythematosus with erythema multiforme-like lesions. Most of the cases have been described in middle-aged women (6). We have described a 53-year-old male.

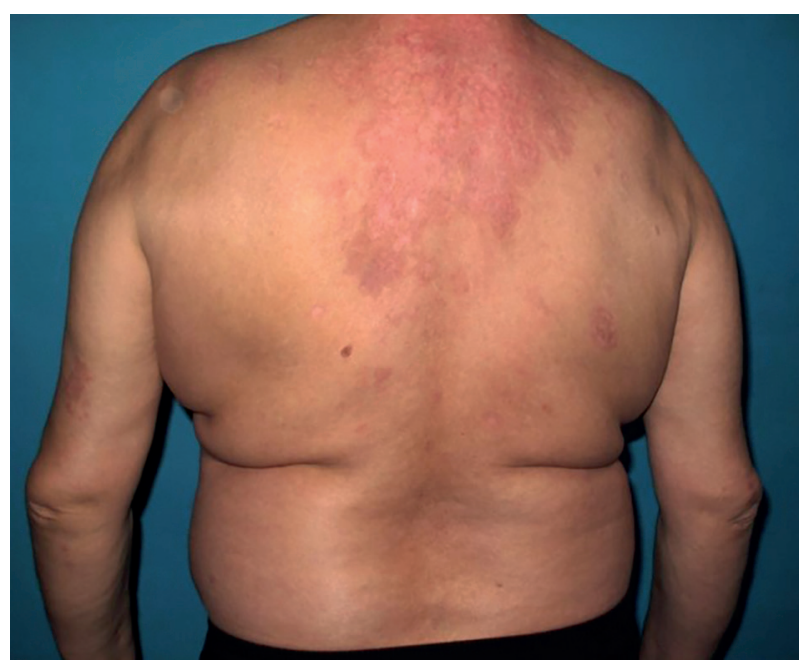

Fig. 3. Postlesional poikilodermic changes upon 1 month of therapy with corticosteroids and antimalarial drugs 
A great controversy exists on the exact taxonomy of Rowell syndrome. Due to inconsistent features the diagnostic criteria have been changed during the years. Rowell et al. coined the initial paradigms: erythema multiforme-like lesions, speckled pattern of ANA, rheumatoid factor, and positive saline extract of human tissue (anti-SJT - now regarded as similar to anti-Ro) (2). Chilblains were included in 1995 (7). In 2000, Zeitouni et al. suggested major (SLE, DLE, or subacute cutaneous LE, EM-like lesions with or without mucosal involvement, and speckled pattern of ANA) and minor (chilblains, anti-Ro/antiLa antibody, and a positive RF) diagnostic criteria. All three major criteria and at least one minor criterion are needed to verify the disease (3). Total antinuclear antibody positivity is the most consistent feature of Rowell syndrome. It is registered in more than $80 \%$ of the patients. Hence, rheumatoid factor is positive in only $41 \%$ of the cases $(8,9)$. Our case meets all Zeitouni's major and most minor criteria with the exception of positive rheumatoid factor.

The most recent Rowell syndrome diagnostic criteria were proposed by Torchia et al. in 2012 (10). The diagnosis requires 4 major and one minor criteria. The major criteria are: (1) presence of chronic CLE (DLE and/or chilblain); (2) presence of EM-like lesions (typical or atypical targets); (3) at least 1 positivity among speckled ANA, anti-Ro/SS-A, and anti$\mathrm{La} / \mathrm{SS}-\mathrm{B}$ antibodies, and (4) negative DIF on lesional EM-like targetoid lesions. The minor criteria include: (1) lack of infectious or pharmacologic triggers; (2) absence of typical EM location (acral and mucosal), and (3) presence of at least 1 additional American College of Rheumatology criterion for diagnosis of SLE besides discoid rash and positive ANA antibodies and excluding photosensitivity, malar rash, and oral ulcers. Our diagnosis is completely compatible with these diagnostic features.

Rowell syndrome has been described with all subtypes of LE (systemic, acute, subacute or discoid) $(2,3,11)$. Our patient shows characteristics of acute systemic lupus erythematosus, verified by the presence of oral ulceration, anemia, ANA positivity with speckled pattern, increased levels of anti-dsDNA antibody and decreased complement levels.

Erythema multiforme is usually triggered by infectious agents, mainly Mycoplasma pneumonia and herpes simplex virus, malignancies or drugs such as antibiotics, tuberculostatics, anti-convulsants, and nonsteroid anti-inflammatory medications (12). In our case, the patient had a history of common cold preceding the onset of skin lesions. Therefore, he had taken a cocktail of anti-inflammatory drugs and antipyretics (ibuprofen, paracetamol and metamizol), thus raising the suspicion of possible drug induction.

SLE and Rowell syndrome respond to a similar treatment. Antimalarials, steroids, azathioprine, dapsone, and cyclosporine have been used with satisfactory results (13-16). Our patient had an excellent response on combined corticosteroid and antimalarial regimen with complete resolution of skin lesions within a month.

\section{CONCLUSION}

Despite its rarity, Rowell syndrome should be suspected in any patient that shows EM lesions in association with LE immunological features. Drug induction in genetically predisposed patients is also possible.

\section{REFERENCES}

1. Scholtz M. Lupus erythematosus acutus disseminatus haemorrhagicus. Arch Derm Syphilol. 1922; 6:466.

2. Rowell NR, Beck JS, Anderson JR. Lupus erythematosus and erythema multiforme-like lesions. A syndrome with characteristic immunological abnormalities. Arch Dermatol. 1963; 88: 176-80.

3. Zeitoni NC, Funaro D, Cloutier RA, Gagné E, Claveau J. Redefining Rowell syndrome. Br J Dermatol. 2000; 142(2):343-6.

4. Antiga E, Caproni M, Bonciani D. The last word on the so-called 'Rowell's syndrome'? Lupus. 2012; 21(6):577-85. doi: 10.1177/0961203311430513.

5. Basu A, Ray Y, Bhowmik P, Rahman M, Goswa$\mathrm{mi}$ RP. SLE in a male patient presented initially as Rowell's syndrome. J Assoc Physic Ind. 2018; 66(1):98-9.

6. Duarte AF, Mota A, Pereira M, Baudrier T, Azevedo F. Rowell syndrome: Case report and review of literature. Dermatol Online J. 2008; 14(3):15.

7. Lee S, Schloss E, Kowichi J. Rowell's syndrome: A case report with subacute cutaneous lupus erythematosus and erythema multiforme. Can J Dermatol. 1995; 7: 807-10. 
8. Lee A, Batra P, Furer V, Cheung W, Wang N, Franks A. Rowell syndrome (systemic lupus erythematosus+erythema multiforme). Dermatol Online J. 2009;15(8):1.

9. Bhat RY, Varna C, Bhatt S, Balachandran C. Rowell syndrome. Ind Dermatol Online J. 2014; 5(Suppl 1):S33-5. doi: 10.4103/2229-5178.144526.

10. Torchia D, Romanellie P, Kerdel FA. Erythema multiforme and Stevens-Johnson syndrome/toxic epidermal necrolysis associated lupus erythematosus. J Am Acad Dermatol. 2012; 67(3):417-21. doi: 10.1016/j.jaad.2011.10.012.

11. Roustan G, Salas C, Barbadillo C, Sanchez Yus E, Mulero J, Simon A. Lupus erythematosus with an erythema multiforme-like eruption. Eur J Dermatol. 2000; 10(6):459-62.

12. Creţu A, Dimitriu $A$, Brănişteanu $D$, Brinişteanu DE. Erythema multiforme-etiopathogenic, clinical and therapeutic aspects. Rev Med Chir Soc Med Nat Iasi. 2015; 119(1):55-61.
13. Khandpur S, Das S, Singh MK. Rowell's syndrome revisited: Report of two cases from India. Int J Dermatol. 2005;44(7):545-9. doi: 10.1111/j.1365-4632.2004.01995.x.

14. Aydogan K, Karadogan S, Balaban Adim S, Tunali S. Lupus erythematosus associated with erythema multiforme: Report of two cases and review of the literature. J Eur Acad Dermatol Venereol. 2005;19(5):621-7. doi: 10.1111/j.1468-3083.2005.01233.x.

15. Torchia D. Rowell syndrome categorized as cutaneous lupus erythematosus subtype. J Am Acad Dermatol. 2012; 67:417-21.

16. Müller CS, Hinterberger LR, Vogt T. Successful treatment of Rowell syndrome using oral cyclosporine A. Int J Dermatol. 2011; 50(8):1020-2. doi: 10.1111/j.1365-4632.2010.04848.x. 